\title{
Fermentation enzymes of Giardia intestinalis, pyruvate: ferredoxin oxidoreductase and hydrogenase, do not localize to its mitosomes
}

Correspondence

Victor V. Emelyanov

vittorio.emelyanov@gmail.com

Received 23 August 2010

Revised 13 January 2011

Accepted 23 February 2011

\section{Victor V. Emelyanovt and Alina V. Goldberg}

Institute for Cell and Molecular Biosciences, Newcastle University, Framlington Place, Newcastle upon Tyne NE2 4HH, UK

\begin{abstract}
It is becoming increasingly clear that the so-called remnant organelles of microaerophilic unicellular eukaryotes, hydrogenosomes and mitosomes, are significantly reduced versions of mitochondria. They normally lack most of the classic mitochondrial attributes, such as an electron transport chain and a genome. While hydrogenosomes generate energy by substrate-level phosphorylation along a hydrogen-producing fermentation pathway, involving iron-sulfur-clustercontaining enzymes pyruvate : ferredoxin oxidoreductase (PFO) and hydrogenase, whether mitosomes participate in ATP synthesis is currently unknown. Both enzymes were recently described in the mitosome-bearing diplomonad Giardia intestinalis, also shown to produce molecular hydrogen. As published data show that giardial PFO is a membrane-associated enzyme, it could be suspected that PFO and hydrogenase operate in the mitosome, in which case the latter would by definition be a hydrogenosome. Using antibodies against recombinant enzymes of $G$. intestinalis, it was shown by Western blot analysis of subcellular fractions and by confocal immunofluorescence microscopy of whole cells that neither PFO nor hydrogenase localize to the mitosome, but are mostly found in the cytosol. The giardial mitosome is known to play a role in iron-sulfur cluster assembly and to contain chaperones Cpn60 and mtHsp70, which assist, in particular, in protein import. In mitochondria, transmembrane potential is essential for this complex process. Using MitoTracker Red and organelle-specific antibodies, transmembrane potential could be detected in the Trichomonas vaginalis hydrogenosome, but not in the $G$. intestinalis mitosome. These results provide further evidence that the Giardia mitosome is one of the most highly reduced mitochondrial homologues.
\end{abstract}

\section{INTRODUCTION}

As described in the literature, the main function of mitochondria is aerobic respiration. Indeed, the ATP yield of aerobic respiration approaches 34 moles per mole of glucose utilized (Saraste, 1999), while a ubiquitous glycolysis (Embden-Meyerhof-Parnas pathway) produces only 2 moles (Dandekar et al., 1999). Along with aerobic mitochondria, which use molecular oxygen as a terminal electron acceptor, versatile mitochondria exist, which also use alternative electron acceptors such as fumarate and oxidized nitrogen compounds (Tielens \& Van Hellemond, 1998; Takaya et al., 1999). Mitochondria are also involved in various metabolic processes, such as iron-sulfur cluster

†Present address: Unité des Rickettsies CNRS-IRD UMR6236-198, Faculté de Médecine, Université de la Méditerranée, 27 Boulevard Jean Moulin, 13385 Marseille, France.

Abbreviations: DIC, differential interference contrast; ER, endoplasmic reticulum; FCCP, 4-(trifluoromethoxy)-phenylhydrazone; IFA, immunofluorescence analysis; PFO, pyruvate : ferrdoxin oxidoreductase. assembly, which is an essential mitochondrial function in yeast (Lill \& Mühlenhoff, 2008).

Mitochondria are convincingly argued to have originated once in evolution from within the alpha subdivision of Proteobacteria (Gray et al., 1998). Numerous phylogenetic data point to the order Rickettsiales of obligate endosymbionts as a taxonomic source of organelles (Emelyanov, 2001, 2003a, b; Fitzpatrick et al., 2006; Williams et al., 2007). Many unicellular eukaryotes, either parasitic or inhabiting niches with little oxygen, were once thought to lack mitochondria. For a long time, these species were classified into the kingdom Archezoa, a paraphyletic group of organisms whose lack of mitochondria was deemed to be a primitive condition (Cavalier-Smith, 1987). Some of them, such as the Archamoeba Entamoeba histolytica and many ciliates, nevertheless branch on phylogenetic trees with eukaryotes, bearing typical mitochondria, thus suggesting that they have a secondarily amitochondriate nature (Embley, 2006; Embley \& Martin, 2006). Among the most enigmatic Archezoa are parabasalids, such as 
Trichomonas vaginalis, and diplomonads, exemplified by Giardia intestinalis, whose phylogenetic position on eukaryotic trees has not been defined unequivocally (Arisue et al., 2005; Keeling et al., 2005; Hampl et al., 2009). Nowadays, a wealth of molecular data has overturned the Archezoa concept in that all the known extant Archezoa, including the two above and some other enigmatic protists, have been shown to contain mitochondrion-derived organelles (Tovar et al., 2003; Embley \& Martin 2006; Hampl et al., 2008). First, it was found that $T$. vaginalis and $G$. intestinalis possess components of the ISC system of iron-sulfur cluster assembly (Tachezy et al., 2001), which is characteristic of mitochondria (Lill \& Mühlenhoff, 2008). Phylogenetic analysis of cysteine desulfurase (IscS), which is an essential component of the ISC, affiliated monophyletic eukaryotes, including these protists, with the order Rickettsiales to the exclusion of free-living alphaproteobacteria, suggesting that diplomonad ancestors possessed bona fide mitochondria (Emelyanov, 2003a). At the same time, a tiny remnant organelle, called a mitosome, was revealed in G. intestinalis, based on localization of the components of the ISC system and their co-localization with bacterial-type chaperones (Tovar et al., 2003; Regoes et al., 2005). The presence and phyletic affiliation of the ISC system with mitochondria as well as its hydrogenosomal localization were also shown for T. vaginalis (Sutak et al., 2004), which was already known to contain a hydrogenosome, an organelle producing molecular hydrogen via the fermentation pathway and generating ATP (Müller, 1993). Second, hydrogenosomes, mitosomes and classic yeast mitochondria were shown to possess a conserved protein import pathway (Bradley et al., 1997; Dolezal et al., 2005; Regoes et al., 2005).

The reduced organelles of mitochondrial origin described above lack the electron transport chain and a genome (Embley \& Martin, 2006). Their energy metabolism relies exclusively on glycolysis and fermentation (Müller, 2003). In hydrogenosomes, pyruvate undergoes oxidative decarboxylation by pyruvate: ferredoxin oxidoreductase (PFO) yielding acetyl-CoA and reduced ferredoxin. Iron-only hydrogenase reoxidizes the latter, coupled to reduction of protons (Müller, 1993, 2003). As in some mitochondria (Van Hellemond et al., 1998), ATP is generated via the concerted action of two enzymes. Acetyl: succinate CoA transferase converts acetyl-CoA to succinyl-CoA. Typically a mitochondrial enzyme, succinyl-CoA synthetase, also known as succinate thiokinase (a component of the Krebs cycle), hydrolyses succinyl-CoA coupled with ATP synthesis (Müller, 1993; van Grinsven et al., 2008). Energy metabolism of hydrogenosomal eukaryotes like Trichomonas was called type II metabolism (Müller, 2003). In Giardia, which possesses type I metabolism (Müller, 2003), acetyl-CoA and reduced ferredoxin are also produced through the action of PFO, but reduced ferredoxin has long been thought to reoxidize via a different route (Townson et al., 1996). This sort of energy production was ascribed to the cytoplasm in agreement with the first data on the cytosolic localization of PFO in Giardia (Lindmark, 1980). It should be noted that neither mitosome nor hydrogen gas production by $G$. intestinalis was known at that time. Remarkably (and of unknown significance), unlike in organisms with hydrogenosomes, acetyl-CoA is directly converted to acetate by the action of ADP-forming acetylCoA synthetase coupled with ATP synthesis in mitosomebearing protists (Sánchez et al., 2000; Hjort et al., 2010).

Subsequently, G. intestinalis PFO was reported to be a membrane-associated enzyme (Ellis et al., 1993; Townson et al., 1996). Subsequently, Giardia was shown to generate molecular hydrogen. The amount of hydrogen produced is one-tenth of that of $T$. vaginalis per cell (Lloyd et al., 2002a), i.e. in view of cell size difference (Müller, 2003) these protists may produce comparable amounts of hydrogen per unit of cell volume. Notably, unlike Trichomonas, G. intestinalis does not possess hydrogenase maturation enzymes, which are normally essential for hydrogenase activity (Pütz et al., 2006). Two genes encoding putative iron-only hydrogenases were also found in a G. intestinalis genome (Morrison et al., 2007). Taken together, these data raise the question of whether PFO and hydrogenase localize to the giardial mitosome, i.e. whether it is a form of hydrogenosome. To address this issue, we cloned and overexpressed Giardia PFO and hydrogenase in Escherichia coli, and raised polyclonal (monospecific) antibodies against purified recombinant proteins. These were used in co-localization experiments and Western blot analysis of subcellular fractions. As organellar markers, antibodies against giardial IscS and Cpn60, which are known to localize to the mitosome (Tovar et al., 2003; Dolezal et al., 2005; Regoes et al., 2005), were used. Using MitoTracker Red and organelle-specific antibodies, we also searched for transmembrane potential in the Giardia mitosome. A similar search for hydrogenosomal potential was also done for $T$. vaginalis to be compared with $G$. intestinalis, given that published data did not identify the organelle itself using hydrogenosome-specific molecular markers (Humphreys et al., 1998).

\section{METHODS}

Growth of G. intestinalis and T. vaginalis. G. intestinalis strain WB trophozoites were propagated in TYI-S-33 medium, which contained $0.05 \%$ bovine bile, antibiotics $\left(0.1 \mathrm{mg}\right.$ penicillin $\mathrm{ml}^{-1}$ and streptomycin) and $10 \%(\mathrm{v} / \mathrm{v})$ heat-inactivated bovine serum (Tachezy et al., 2001). For immunofluorescence analysis (IFA), the cells were grown in either $2 \mathrm{ml}$ vials or $7 \mathrm{ml}$ plastic tubes to form a subconfluent layer (48 h at $37^{\circ} \mathrm{C}$ ). For preparative growth, a $2 \mathrm{ml}$ plastic vial with a confluent layer was chilled in an ice bath for $20 \mathrm{~min}$ and the material $\left(2 \times 10^{6}\right.$ cells $\left.\mathrm{ml}^{-1}\right)$ was transferred to a $50 \mathrm{ml}$ sterile flask containing complete TYI-S-33 medium. After 48 h of growth, when the confluent layer was formed, the flask was chilled as above and its contents (2$3 \times 10^{6}$ cells ml $^{-1}$ ) were transferred to two triple flasks (Nunc, approximately 0.751 each). A confluent layer of Giardia cells was typically reached after $48-60 \mathrm{~h}$ incubation at $37{ }^{\circ} \mathrm{C}$. After cooling, the cells $\left(1.5-2 \times 10^{6}\right.$ cells $\left.\mathrm{ml}^{-1}\right)$ were pelleted at $900 \mathrm{~g}$ for $8 \mathrm{~min}$ at $4{ }^{\circ} \mathrm{C}$ followed by washing twice with $10 \mathrm{~min}$ centrifugations with HSDP buffer composed of $10 \mathrm{mM}$ HEPES-KOH pH 7.2, $0.25 \mathrm{M}$ sucrose, 
$4 \mathrm{mM}$ DTT, $0.1 \mathrm{mM}$ PMSF, $10 \mu \mathrm{g}$ leupeptin $\mathrm{ml}^{-1}$ and $50 \mu \mathrm{g} N$-tosyl-Llysine chloromethyl ketone $\mathrm{ml}^{-1}$. Finally, the cells were suspended in $6 \mathrm{ml} \mathrm{HSDP}$ and snap-frozen in liquid nitrogen as $3 \times 2 \mathrm{ml}$ aliquots. T. vaginalis (strain G3) cells were grown in modified Diamond's medium at $37{ }^{\circ} \mathrm{C}$ (Hirt et al., 2003) in $7 \mathrm{ml}$ plastic tubes, till cell density reached $5 \times 10^{5}$ c.f.u. $\mathrm{ml}^{-1}$.

Generation of antisera to G. intestinalis proteins. G. intestinalis PFO (GenBank accession no. L27221) and Fe-only hydrogenase (AF242293) genes were amplified by PCR from genomic DNA using designed primers (sequences available from the authors on request) and cloned into expression vector pET-16b (Novagen). The constructs were confirmed by sequencing and transformed into $E$. coli BL21 cells. Full-length PFO and hydrogenase were expressed as recombinant His-tagged proteins, and the resulting inclusion bodies were partially purified by using BugBuster reagent (Novagen). The proteins were then purified by a preparative PAGE. Hydrogenase $(0.25 \mathrm{mg})$ and PFO $(1.5 \mathrm{mg})$ were used for the commercial production of rat and rabbit polyclonal antisera (Eurogentec), respectively. Cloning and production of monospecific antibody against Giardia Cpn60 were performed in a similar way.

Measurement of organellar transmembrane potential. Subconfluent cultures of $G$. intestinalis in three $2 \mathrm{ml}$ sterile vials were treated (protected from light) as follows. 4-(Trifluoromethoxy)phenylhydrazone (FCCP, Sigma-Aldrich) was added from $80 \mathrm{mM}$ stock solution in acetone to one tube at a final concentration of $100 \mu \mathrm{M}$, followed by $1 \mathrm{~h}$ incubation at $37^{\circ} \mathrm{C}$. MitoTracker Red was added from $40 \mu \mathrm{M}$ stock solution in DMSO to each tube at a final concentration of $0.5-1.5 \mu \mathrm{M}$, and incubation was continued for $1 \mathrm{~h}$. Tubes were chilled in an ice bath for $20 \mathrm{~min}$, centrifuged at $4{ }^{\circ} \mathrm{C}$ for $4 \mathrm{~min}$ at $900 \mathrm{~g}$, and washed once with ice-cold PBS with recentrifugation. All subsequent treatments were carried out at room temperature. Each sample was suspended in $0.5 \mathrm{ml} \mathrm{PBS}$, and $0.5 \mathrm{ml} 6 \%$ formaldehyde in PBS was added to each tube. Fixation was continued at room temperature for 30-40 min followed by washing with PBS once with centrifugation for $4 \mathrm{~min}$ at $900 \mathrm{~g}$. The cells were incubated for $5 \mathrm{~min}$ in $0.5 \mathrm{ml}$ PBS containing $0.1 \mathrm{M}$ glycine, and permeabilized for $20 \mathrm{~min}$ in $0.5 \mathrm{ml}$ PBS containing $2 \%$ BSA and $0.2 \%$ Triton X-100. After washing with PBS with centrifugation as above, the cells were suspended in $0.2 \mathrm{ml} \mathrm{PBS}$ and attached to poly-L-lysine-coated slides of eight-well glass slides (Nunc) for $1 \mathrm{~h}$. The cells were then blocked by using 2\% BSA for $2.5 \mathrm{~h}$ (Regoes et al., 2005; Stefanic et al., 2006). Rabbit anti-IscS (1:400) or anti-Cpn60 (1:100), and anti-rabbit secondary antibody conjugated to Alexa-fluor 488 (Molecular Probes) were diluted in PBS containing 2\% BSA and $0.2 \%$ Triton X-100. In control experiments with non-organellar markers, rabbit anti-PFO $(1: 150)$ or rat anti-hydrogenase $(1: 200)$ was used. Incubation with each antibody proceeded for $1 \mathrm{~h}$, with three washes between incubations with PBS containing $0.5 \%$ BSA and $0.05 \%$ Triton X-100 (Stefanic et al., 2006). Finally, the samples were washed with PBS, mounted with DAPI-containing anti-fade mounting reagent (Vectashield), and observed under a laser scanning confocal microscope (Leica, TCS SP2 UV) fitted with a $\times 63$ objective (Plan Apo, $1.32 \mathrm{nA}$ ). Images were collected using LCS V2.61 software (Leica Microsystems) and processed with Adobe Photoshop CS2. After treatment with FCCP and MitoTracker Red ( $0.5 \mu \mathrm{M}$ final concentration) or MitoTracker Red alone (as above but in $7 \mathrm{ml}$ plastic tubes), T. vaginalis cells were pelleted at $350 \mathrm{~g}$ for $5 \mathrm{~min}$ at $4{ }^{\circ} \mathrm{C}$, washed once with potassium PBS (PPBS, $10 \mathrm{mM}$ potassium phosphate $\mathrm{pH} 7.2$ and $0.15 \mathrm{M} \mathrm{NaCl}$ ), and fixed for $15 \mathrm{~min}$ at room temperature in the same buffer containing 3\% formaldehyde. After two washes with PPBS with centrifugations as above, the cells were permeabilized for $10 \mathrm{~min}$ at room temperature in PPBS with $0.2 \%$ Triton X-100. The cells were washed once with PPBS, suspended in the same buffer and attached for $1 \mathrm{~h}$ to glass slides, as for Giardia. The samples were treated with $0.1 \mathrm{M}$ glycine, and blocked in $5 \%$ BSA for $0.5 \mathrm{~h}$. Primary and secondary antibodies were diluted in
PPBS containing 2\% BSA. Rabbit antibody against hydrogenosomal malic enzyme and monoclonal antibody against non-organellar marker $\alpha$-tubulin (Delgado-Viscogliosi et al., 1996) were diluted 1:500 and $1: 1000$, respectively. T. vaginalis cells were washed three times with PPBS after $1 \mathrm{~h}$ incubations with antibodies (Hirt et al., 2003). The samples were mounted for IFA as above.

Immunolocalization of PFO and hydrogenase in G. intestinalis. Trophozoites of G. intestinalis were grown in $7 \mathrm{ml}$ plastic tubes and prepared for confocal microscopy essentially as described above. Incubation with primary antibody (rabbit anti-IscS or anti-Cpn60) was followed by incubation with anti-rabbit secondary antibody conjugated to Alexa-fluor 594 (Molecular Probes), and subsequent treatment with a second (rat anti-hydrogenase) primary antibody was followed by incubation with anti-rat secondary antibody conjugated to Alexa-fluor 488 (Molecular Probes). As anti-PFO antibody was raised in rabbits, we first purified anti-IscS immunoglobulins using the Melon IgG spin purification kit (Pierce) and then biotinylated these with the EZ-link Sulfo NHS-LC biotinylation kit (Pierce) to enable detection via the streptavidin conjugate (streptavidin-Alexa 488, Invitrogen).

Purification of G. intestinalis mitosomes. All steps were done at $4{ }^{\circ} \mathrm{C}$ unless otherwise stated. Cell suspension $(2 \mathrm{ml})$ from liquid nitrogen (see above) was thawed, and $5 \mathrm{ml}$ HSDP buffer was added, followed by ultrasound treatment for $15 \mathrm{~s}$ in an ice bath. The completeness of cell breakage was monitored by light microscopy. The suspension was centrifuged at $3000 \mathrm{~g}$ for $20 \mathrm{~min}$ to remove nuclei and cell debris. An aliquot for electrophoresis and Western blot anaylsis was saved as the S3 fraction. Post-nuclear supernatant $(7 \mathrm{ml})$ was centrifuged at $5600 \mathrm{~g}$ for $0.5 \mathrm{~h}$. The supernatant was ultracentrifuged at $50000 \mathrm{~g}$ for $1.5 \mathrm{~h}$. The resulting pellet was resuspended in $7 \mathrm{ml}$ HSDP and resedimented at $100000 \mathrm{~g}$ for $1 \mathrm{~h}$. The resulting pellet was resuspended in $0.7 \mathrm{ml} \mathrm{HSDP}$, and an aliquot was saved as P50. The rest was layered on a $10-35 \%(\mathrm{w} / \mathrm{v})$ linear Nycodenz (Axis-Shield) density gradient, prepared with HSDP in an Ultra-Clear centrifuge tube, and ultracentrifuged at $155000 \mathrm{~g}$ for $2 \mathrm{~h}$. Material of the second band from the top (approx. $16 \%$ Nycodenz) was diluted seven times with HSDP and pelleted at $175000 \mathrm{~g}$ for $1 \mathrm{~h}$. The supernatant obtained above after the centrifugation at $50000 \mathrm{~g}$ (S50) was centrifuged in parallel, and the supernatant was saved as S175. Pelleted material of fraction 2 of the linear Nycodenz gradient was resuspended in $0.2 \mathrm{ml} \mathrm{HSDP}$, loaded onto a discontinuous 15, 17.5 and $20 \%(\mathrm{w} / \mathrm{v})$ Nycodenz gradient, and ultracentrifuged at $199000 \mathrm{~g}$ for $1.5 \mathrm{~h}$. Material from the $15 / 17.5 \%$ interphase was diluted seven times with HSDP, pelleted at $150000 \mathrm{~g}$ for $1 \mathrm{~h}$, loaded onto a second discontinuous Nycodenz gradient (13.5, $16,18.5 \%$ ) and centrifuged again at $150000 \mathrm{~g}$ for $1 \mathrm{~h}$. Material from the $13.5 / 16 \%$ interphase was diluted seven times with HSDP, pelleted at $150000 \mathrm{~g}$ for $1 \mathrm{~h}$, resuspended in $0.1 \mathrm{ml} \mathrm{HSDP}$, divided into four aliquots of $25 \mu$ l, quickly frozen in liquid nitrogen, and stored at $-80{ }^{\circ} \mathrm{C}$ as the mitosomal fraction. All fractions were analysed by Western blot using antibodies against mitosomal markers IscS and Cpn60. Contamination of mitosomal material with endoplasmic reticulum (ER) was checked by using monoclonal antibody against the protein disulfide isomerase-2 (PDI-2) (Stefanic et al., 2006).

Fractionation of giardial cells by differential centrifugation. All procedures were done essentially as described above. S3 supernatant was centrifuged in an $80 \mathrm{Ti}$ rotor at $5600 \mathrm{~g}$ for $0.5 \mathrm{~h}$, and the pellet (P6) washed with $7 \mathrm{ml} \mathrm{HSDP}$ with recentrifugation, while the supernatant (S6) was centrifuged for $1 \mathrm{~h}$ at $50000 \mathrm{~g}$. Subsequent ultracentrifugations at 100000 and $190000 \mathrm{~g}$ were carried out for $1 \mathrm{~h}$, and each pellet was washed with recentrifugation to remove trapped material. To keep the correct supernatant/pellet ratio, $10 \mu \mathrm{l}$ of each supernatant $(7 \mathrm{ml})$ and $1 \mu \mathrm{l}$ of each resuspended pellet $(0.7 \mathrm{ml})$ were taken for SDS-PAGE followed by immunoblotting. Monoclonal antibodies against PDI-2 (membrane-associated ER enzyme) and PDI-1 (enzyme of ER lumen) served as ER markers (Stefanic et al., 2006). 
SDS-PAGE and Western blotting. Proteins of whole cells, centrifugation pellets and supernatants, and partially purified mitosomes were separated by SDS-PAGE, as described by Laemmli (1970). They were transferred from gels onto nitrocellulose membranes using a semi-dry transfer unit, followed by staining with Ponceau S (Sigma, diluted five times with water). Membranes were processed following standard protocols. They were destained, blocked with $5 \%$ skimmed milk (Merck), and incubated with the anti-IscS $(1: 6000)$, rabbit antiCpn60 (1:200), rabbit anti-PFO (1:1000), rat anti-hydrogenase $(1: 300)$, mouse anti-PDI-2 $(1: 400000)$ or anti-PDI-1 $(1: 200000)$ antisera, followed by secondary anti-rabbit, anti-rat or anti-mouse antibodies conjugated to peroxidase (Sigma). The blots were developed using enhanced chemiluminescence (Amersham Biosciences).

\section{RESULTS}

\section{Non-mitosomal localization of G. intestinalis fermentation enzymes}

Using laser scanning confocal immunofluorescence microscopy, co-localization of hydrogenase with IscS
(Fig. 1a) and Cpn60 (not shown) was not found. Similarly, PFO did not co-localize with IscS (Fig. 1b), and PFO and hydrogenase did not co-localize with each other (Fig. 1c). Both PFO and hydrogenase are seen as rather scarce cytosolic enzymes, which may be a reason for the absence of co-localization signal. Notably, both enzymes are also partially present in very small aggregates, which may represent some unknown organelles. At any rate, neither enzyme localized to clearly visible mitosomes (Fig. 1a, b).

In immunoblot analysis, both enzymes were detected in whole cells and high-speed supernatants of the cell lysate, but not in the $50000 \mathrm{~g}$ pellet and Nycodenz density gradient fractions, which are highly enriched in the mitosome (Fig. 2a, b). When additional subcellular fractions were prepared using a higher centrifugation speed, PFO and (to a much lesser extent) hydrogenase were shown to partition between the $190000 \mathrm{~g}$ pellet and supernatant; however, neither was found in the $100000 \mathrm{~g}$ (a)

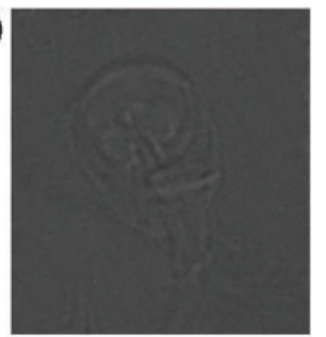

DIC

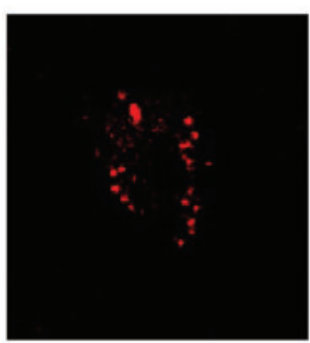

Anti-IscS

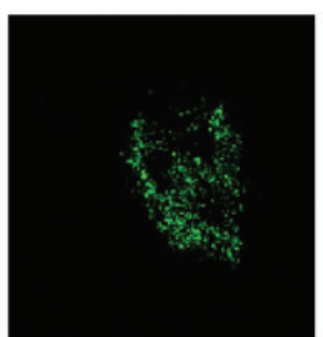

Anti-hydrogenase

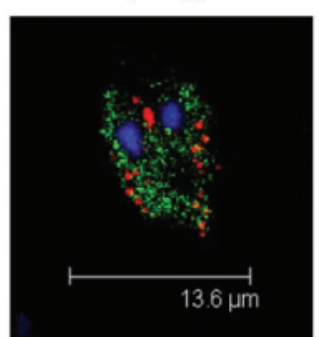

Merge + DAPI (b)

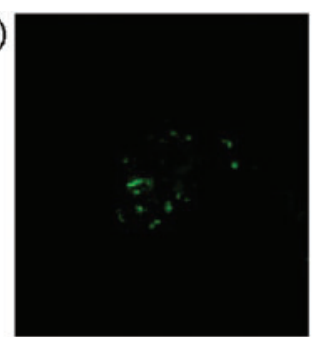

Anti-IscS

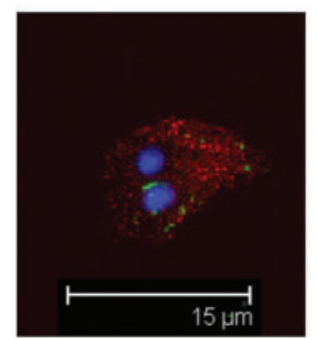

Merge + DAPI

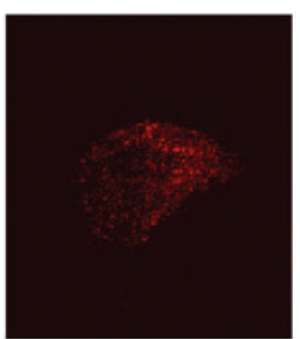

Anti-PFO

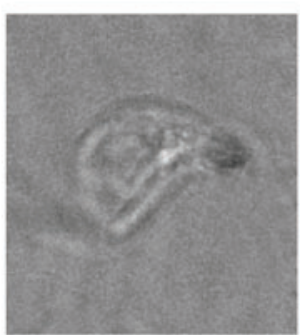

DIC

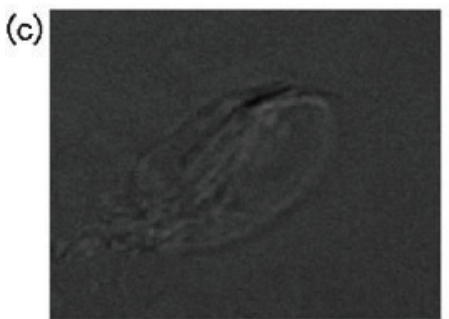

DIC

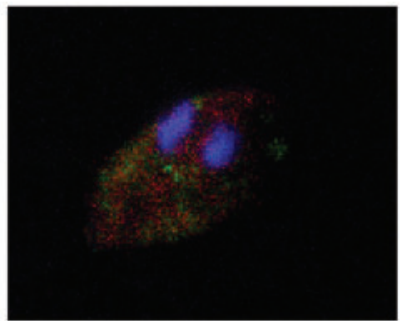

Merge + DAPI

Fig. 1. Cellular localization of G. intestinalis Fe-only hydrogenase (a) and PFO (b), and their co-localization (c) in situ using fluorescence confocal microscopy. (a) The polyclonal antiserum against mitosomal marker cysteine desulfurase lscS (red) labels discrete structures reminiscent of Giardia mitosomes within the cytosol (Tovar et al., 2003; Dolezal et al., 2005; Regoes et al., 2005), while the rat polyclonal antiserum raised against hydrogenase (green) shows diffuse and patchy distribution. No colocalization is observed. (b) Biotinylated antibody (1:50) to IscS (green) does not co-localize with PFO (red). (c) PFO (red) and hydrogenase (green) do not co-localize with each other. The diplomonad's nuclei are stained with DAPI (blue). Differential interference contrast (DIC) images of the same fields are also shown. 
(or less) pellet (Fig. 3). This observation may reconcile earlier data, showing that PFO localizes to the cytosol in one case (Lindmark, 1980) or that $70 \%$ of PFO associates with membrane fractions in other studies (Ellis et al., 1993; Townson et al., 1996). It is not clear from which cellular membrane the structures sedimentable at $190000 \mathrm{~g}$ are derived, but it is unlikely to be the ER, given that the 50000 $g$ pellet contains marker proteins for ER but no signal for PFO and hydrogenase. Accordingly, P190 enriched in PFO contains only trace amounts of ER marker PDI-2 (Fig. 3). This partial membrane localization of fermentation enzymes may be consistent with the observation of small aggregates in IFA (see above). PFO and hydrogenase may, however, be contained in different structures, given that P190 contains only trace amounts of hydrogenase but $50 \%$ PFO. This fact may explain the absence of co-localization of PFO and hydrogenase. In contrast with fermentation enzymes, mitosomal markers IscS (Fig. 2c) and Cpn60 (Figs $2 \mathrm{c}$ and 3 ) were detected in the $50000 \mathrm{~g}$ pellet and partially purified mitosome.

Taken together, these data strongly suggest that the mitosome of $G$. intestinalis is not a compartment of $\mathrm{H}_{2}$ evolving fermentation, which seems to proceed mostly in the cytoplasm instead.

\section{If present, the mitosomal transmembrane potential of Giardia is below the detection limit}

While PFO and hydrogenase are non-mitosomal enzymes, the giardial mitosome still needs to import at least the components of the ISC system and chaperones mtHsp70 and Cpn60 (Dolezal et al., 2005; Regoes et al., 2005). Many preproteins imported by classic mitochondria contain amino-terminal cleavable presequence, mitochondriontargeting sequence, with basic residues at the extreme $\mathrm{N}$ terminus (Mokranjac \& Neupert, 2008). However, it is not very clear whether positive charge is required for electrophoretic transport across the polarized inner membrane or for binding to a specific receptor, or both.
A transmembrane potential is known to be a prerequisite for import of most mitochondrial proteins in yeast (Mokranjac \& Neupert, 2008).

We undertook a search for organellar potential by confocal fluorescence microscopy using MitoTracker Red and organelle-specific antibodies. MitoTracker Red, also called CMXRos (Invitrogen), was chosen because it was shown to be a more reliable tracer of potential in classic mitochondria compared with many other fluorescent dyes and to be fixed better than, for example, rhodamine 123 (Pendergrass et al., 2004). As high transmembrane potential is not expected in mitochondrion-derived organelles, control experiments involved FCCP to depress any putative potential, to be compared with samples without protonophore treatment. For the same comparison purpose, other controls included MitoTracker and antibodies against nonorganellar proteins. The data presented show that the hydrogenosome of $T$. vaginalis does possess transmembrane potential (Fig. 4a). Even after pretreatment of Trichomonas culture with FCCP, some co-localization of malic enzyme (hydrogenosomal marker) with MitoTracker Red may be seen (Fig. 4b). As expected, no co-localization was observed when using antibody against the cytosolic marker tubulin (not shown).

Using antibodies against mitosomal markers IscS (Fig. 5) and Cpn60 (not shown), no co-localization of these antibodies with MitoTracker was shown, regardless of whether Giardia cells were pretreated with protonophore (Fig. 5b) or not (Fig. 5a). A similar pattern was observed when using antibodies against non-mitosomal enzymes PFO (Fig. 5c) or hydrogenase (not shown). An inference here is that even such a sensitive method as fluorescence microscopy failed to reveal transmembrane potential in the giardial mitosome. Even if some potential exists, it is below the detection limit of the technique used. As giardial plasma membrane, like plasma membrane of other eukaryotic cells, contains transmembrane potential (Biagini et al., 2000), MitoTracker Red is evenly distributed in the cytosol. Its absence in the cytoplasm of $T$. vaginalis
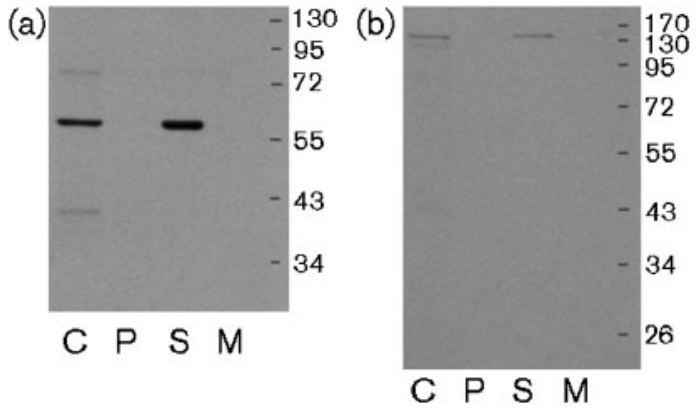

Fig. 2. Western blot analysis of fractionated G. intestinalis cells. Giardial proteins were separated by $10 \%$ SDS-PAGE and transferred onto nitrocellulose membrane. Membranes were incubated with antisera to (a) hydrogenase, (b) PFO and (c) IscS and Cpn60. G. intestinalis whole cells (C), the pellet after centrifugation of a cleared lysate at $50000 \mathrm{~g}$ $(P)$, the supernatant after ultracentrifugation of $50000 \mathrm{~g}$ supernatant at $175000 \mathrm{~g}(\mathrm{~S})$ and the mitosome-rich fraction after purification via three consecutive Nycodenz density gradients (M) are shown. Molecular masses (in $\mathrm{kDa}$ ) of protein markers are indicated. 


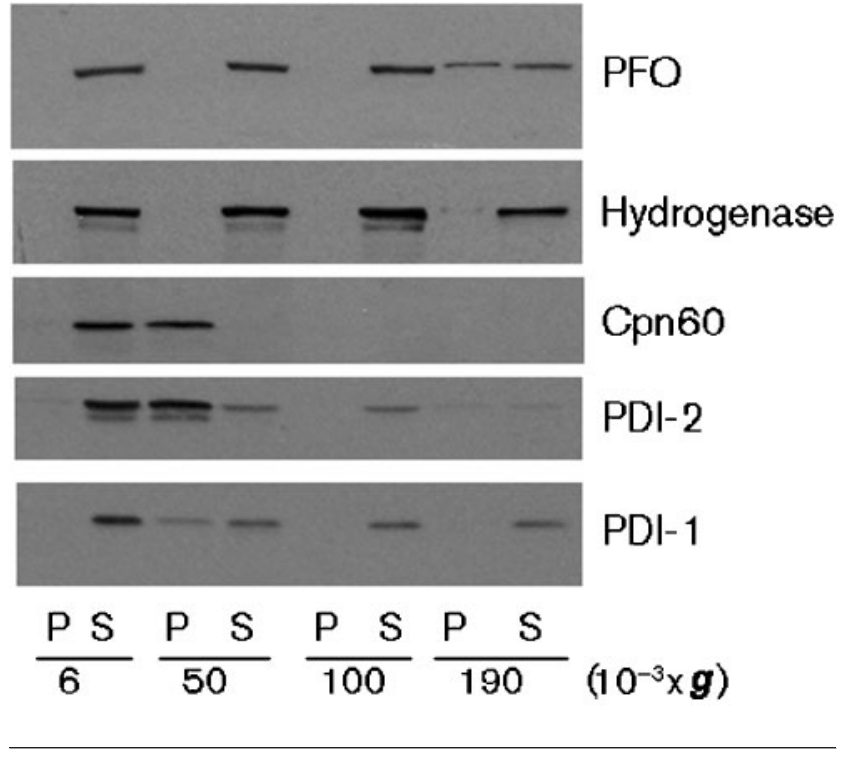

Fig. 3. Differential centrifugation of cleared giardial lysates. Immunoblot of the centrifugation pellets and supernatants with various antibodies. Names of the detected proteins with antibodies are given to the right. P, Pellet; $\mathrm{S}$, supernatant. Numbers indicate $10^{-3} \times g$.

(Fig. 4) may be due to the relatively high MitoTracker Red concentration in the hydrogenosome, so that cytosolic content represents an actually invisible background. As fluorescent dye does not penetrate the giardial mitosome, unlike the hydrogenosome, only the cytosolic background is seen (Fig. 5).

\section{DISCUSSION}

\section{The G. intestinalis remnant organelle is not a hydrogenosome}

In light of the fact that $G$. intestinalis produces molecular hydrogen (Lloyd et al., 2002a) and PFO was reported to be a membrane-associated enzyme (Ellis et al., 1993; Townson et al., 1996), we readily suggested that its mitochondrionderived organelle is nothing but a hydrogenosome. However, this appears not to be the case: the mitosome does not contain fermentation enzymes.

Thus, while both the $G$. intestinalis mitosome and Trichomonas hydrogenosome possess the same mitochondrion-derived iron-sulfur cluster assembly system (Tachezy et al., 2001), they differ in the localization of the iron-sulfur-cluster-containing fermentation enzymes PFO and hydrogenase. It is indeed puzzling why $\mathrm{H}_{2-}$ evolving fermentation proceeds in hydrogenosomes, including those of Trichomonas (Müller, 2003), but not in giardial mitosomes.

As $\mathrm{PFO}$, generating acetyl-CoA, does not localize in the mitosome, and ATP is generated through the action of ADP-forming acetyl-CoA synthetase (Sánchez et al., 2000), it is highly unlikely that the giardial mitosome produces ATP. In that case, the latter enzyme would have to be localized in the mitosome, and the mitosome would, in turn, have to import acetyl-CoA from the cytoplasm. How giardial mitosomes make or take up ATP, which is obviously required for mtHsp70 and Cpn60 functions, remains to be answered, as Giardia appears to lack an ATP/ ADP carrier of any type (Hjort et al., 2010). In contrast,

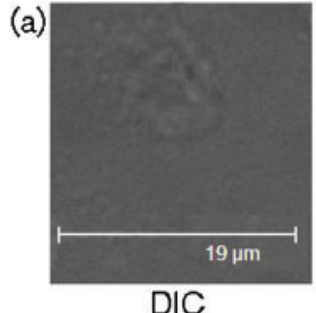
DIC

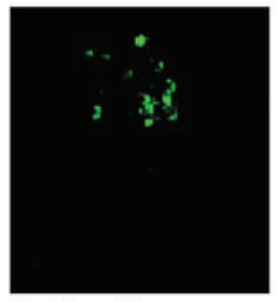

Anti-malic enzyme

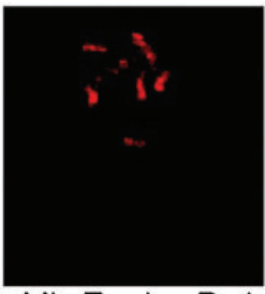

MitoTracker Red

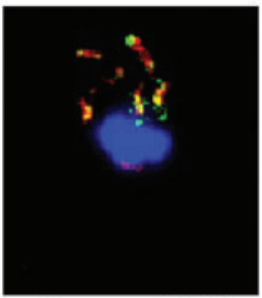

Merge + DAPI

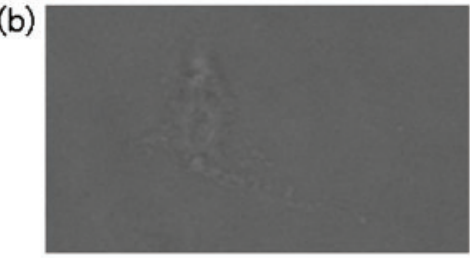

DIC

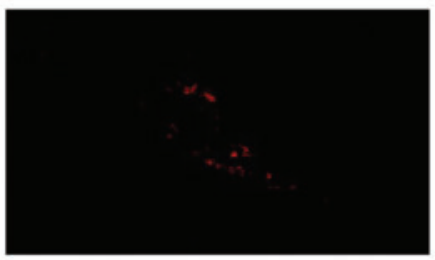

MitoTracker Red

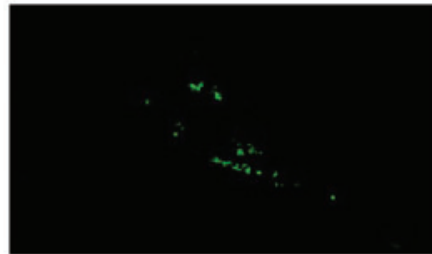

Anti-malic enzyme

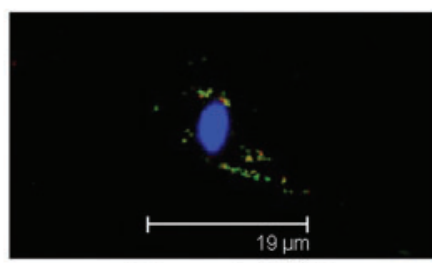

Merge + DAPI

Fig. 4. Detection of $T$. vaginalis hydrogenosome transmembrane potential by using confocal microscopy. (a) Cells pretreated with MitoTracker Red $(0.5 \mu \mathrm{M})$ were bound to a coverslip and incubated with rabbit polyclonal antibody to malic enzyme (organellar marker) followed by incubation with anti-rabbit antibody conjugated to Alexa-fluor 488. (b) Before treatment with MitoTracker Red the cells were incubated for $1 \mathrm{~h}$ in the presence of $0.1 \mathrm{mM}$ FCCP. For other details see the legend to Fig. 1. 
(a)
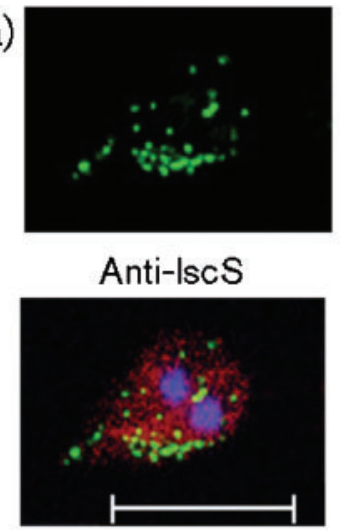

Merge + DAPI

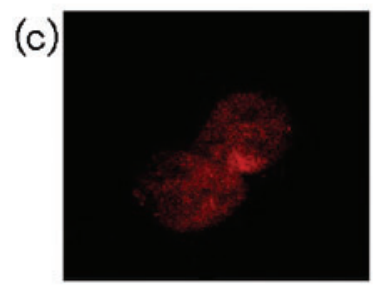

MitoTracker Red

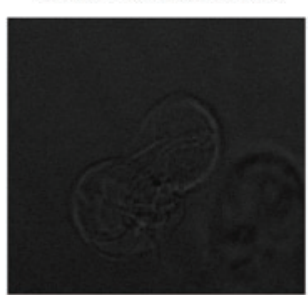

DIC

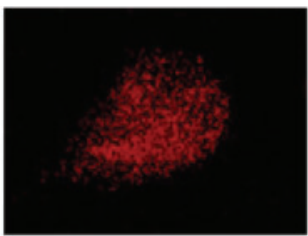

MitoTrackerRed

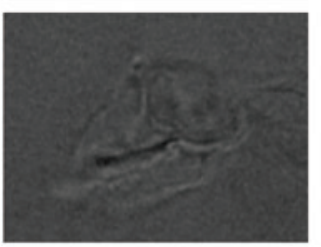

DIC

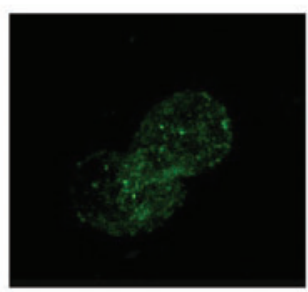

Anti-PFO

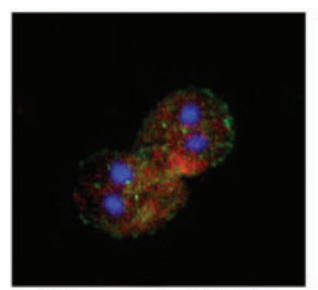

Merge + DAPI (b)

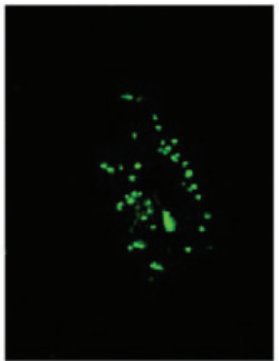

Anti-lscS

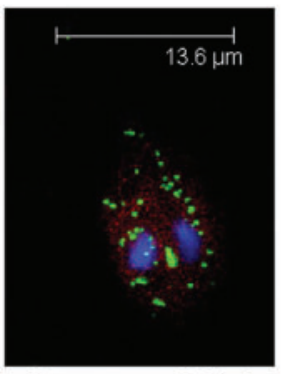

Merge + DAPI

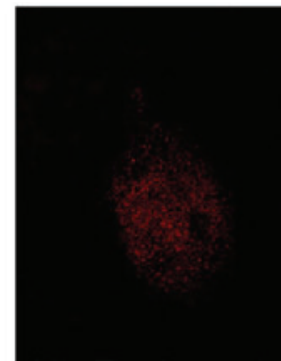

MitoTrackerRed

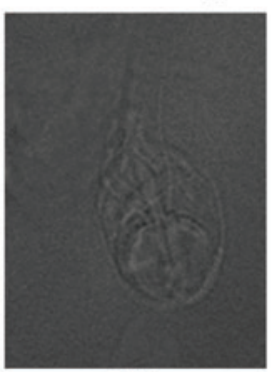

DIC

Fig. 5. IFA measurement of transmembrane potential in the G. intestinalis mitosome. (a) Anti-IscS serum (green) was used to detect mitosomes within the cells prestained with MitoTracker Red. (b) Before addition of MitoTracker Red, the cells were incubated with FCCP $(0.1 \mathrm{mM})$. (c) Along with MitoTracker Red, anti-PFO serum (green) was used as a non-mitosomal marker. For other details see the legend to Fig. 1.

Entamoeba histolytica, another free-living microaerophilic protist, possesses an ATP/ADP carrier of mitochondrial type (Chan et al., 2005), and the PFO and hydrogenase of this organism were suggested not to function in the mitosome (Aguilera et al., 2008).

Thus far, the only known function of the G. intestinalis mitosome remains to be an involvement in iron-sulfur cluster assembly by means of a mitochondrion-derived ISC system (Hjort et al., 2010). Archamoebae (Entamoeba histolytica and Mastigamoeba balamuthi) are known to possess an NIF system of iron-sulfur cluster assembly instead of an organelle-associated ISC system, which was suggested to have been laterally transferred from epsilonproteobacteria and localizes in both the cytosol and the mitosome (Gill et al., 2007; Maralikova et al., 2010). This raises the question of why Giardia retained mitochondrial-like ISC (or Archamoebae acquired the NIF system to replace ISC), especially given that its important iron-sulfur-containing enzymes, PFO and hydrogenase, do not function in the mitosome, as is also suggested for Entamoeba (Aguilera et al., 2008). The simplest explanation is that $G$. intestinalis or even diplomonads as a whole had no opportunity for a non-orthologous replacement of ISC machinery with the much simpler (Maralikova et al., 2010) NIF system. Another possibility is that, unlike amoebae, $G$. intestinalis could have been unable to direct components of the NIF system into the mitosome, that is of course if iron-sulfur cluster assembly is needed in the giardial mitosome, as it probably takes place in the Entamoeba mitosome containing the iron-sulfur protein rubrerythrin (Maralikova et al., 2010).

As anaerobic energy metabolism appears to be quite ubiquitous among eukaryotes (Embley \& Martin, 2006) and supposedly relates to eukaryogenesis itself (Martin \& Müller, 1998; Emelyanov, 2003c, 2007; Embley \& Martin, 2006), the evolutionary origins of eukaryotic PFO and hydrogenase deserve much attention. Phylogenetic data 
based on PFO and iron-only hydrogenase sequences have been published on numerous occasions. While phylogenetic analysis of PFO typically shows a monophyly of eukaryotes with the bacterial donor taxon currently being uncertain, hydrogenase has an apparently polyphyletic distribution (Horner et al., 1999; Embley et al., 2003; Embley, 2006; Hampl et al., 2008; Hug et al., 2010). The hypotheses thought to be most relevant for the origin of anaerobic energy metabolism are based on syntrophydriven endosymbiosis (Martin \& Müller, 1998). The most parsimonious and popular scenario, the so-called hydrogen hypothesis, speculates that $\mathrm{H}_{2}$-evolving fermentation, like glycolysis, another anaerobic process, has been acquired by an archaeal host from an alphaproteobacterial endosymbiont which has given rise to mitochondria (Martin \& Müller, 1998). However, fermentation (e.g. Hug et al., 2010) and glycolysis (Emelyanov, 2003c, 2007) enzymes do not affiliate eukaryotes with alphaproteobacteria, unlike aerobic respiration (Emelyanov, 2003b). Furthermore, the hydrogen hypothesis suffers from many other drawbacks (Emelyanov, 2007). A more complex scenario of eukaryogenesis, called fusion theory, invokes two consecutive endosymbioses at the origin of eukaryotes (Gupta, 1998; Emelyanov, 2003c). In these models, the mitochondrial origin has been preceded by an endosymbiosis of a member of the archaea in a fermenting eubacterium, which could be a donor of PFO and hydrogenase. It should be noted, however, that phylogenetic data did not identify any particular eubacterial group that could be the donor taxon of fermentation enzymes (see references above), while analysis of some glycolytic enzymes and valyl-tRNA synthetase pointed to beta- and gammaproteobacteria as possible eubacterial partners of the first endosymbiotic event (Emelyanov, 2003c, 2007).

\section{In contrast with the Trichomonas hydrogenosome, the G. intestinalis mitosome is not a charged organelle}

Mitochondrion-targeting sequences of classic organelles are known to contain positively charged amino acid residues at the extreme $\mathrm{N}$ terminus (Mokranjac \& Neupert, 2008). Recent examination of Giardia mitosomal and Trichomonas hydrogenosomal preproteins showed that: (i) most mitosomal precursors lack cleavable presequences, and when present, they do not contain terminal basic residues and (ii) some hydrogenosomal precursors do contain positively charged amino acid residues at the $\mathrm{N}$ terminus (Smíd et al., 2008). These observations raised the question of whether remnant organelles of these organisms possess transmembrane potential. Earlier data suggested that the hydrogenosome of $T$. vaginalis is a charged organelle. Rhodamine 123 and cationic fluorescent dyes have been used to address the question (Humphreys et al., 1998), but an organelle as such has not been identified in situ independently using hydrogenosome-specific markers. It was also shown that in vitro import of proteins into the T. vaginalis hydrogenosome is blocked by the protonophore $m$-chlorophenylhydrazone, although at a very high $(0.6 \mathrm{mM})$ concentration (Bradley et al., 1997). Rhodamine 123 was shown to stain some structures within the giardial cytosol, but these are too large in size and too scarce in number to be mitosomes, which was unknown at that time (Lloyd et al., 2002b).

In the present work, a co-localization approach was used, i.e. the organelle was identified independently of organellepenetrating dye (here MitoTracker Red) using antibodies specific to organellar proteins. While transmembrane potential could easily be detected in the Trichomonas hydrogenosome, it could not be revealed in the $G$. intestinalis mitosome under similar conditions. It is notable that this observation correlates with the absence of positively charged amino acid residues in presequences of mitosome-targeted proteins (Smíd et al., 2008). It is not clear why MitoTracker Red did not reveal the structures in the giardial cytoplasm that were observed by Lloyd et al. (2002b) using rhodamine 123 (see above), and it is also not known what these large structures are. It should be pointed out that, in our work, MitoTracker Red showed punctate distribution in some cases (Fig. 5). It is unclear whether this is an artefact of the fixation process or staining of some new tiny organelles, e.g. those described recently in the $G$. intestinalis cytoplasm as tubulovesicular structures (Abodeely et al., 2009).

\section{ACKNOWLEDGEMENTS}

V.V.E. was supported by the Marie Curie Incoming International Fellowship FP6 (grant MIF1-CT-2006 039819). Thanks are due to Jan Tachezy and Adrian B. Hehl, who provided antibodies against giardial IscS and Trichomonas malic enzyme, and monoclonal antibodies to Giardia PDI-1 and PDI-2, respectively. We also thank Trevor Booth for help with IFA. We are much indebted to Professor T. Martin Embley, in whose laboratory this study was carried out. Thanks are also due to Martin Embley and Robert P. Hirt for fruitful discussions.

\section{REFERENCES}

Abodeely, M., DuBois, K. N., Hehl, A., Stefanic, S., Sajid, M., DeSouza, W., Attias, M., Engel, J. C., Hsieh, I. \& other authors (2009). A contiguous compartment functions as endoplasmic reticulum and endosome/lysosome in Giardia lamblia. Eukaryot Cell 8, 1665-1676.

Aguilera, P., Barry, T. \& Tovar, J. (2008). Entamoeba histolytica mitosomes: organelles in search of a function. Exp Parasitol 118, 10-16.

Arisue, N., Hasegawa, M. \& Hashimoto, T. (2005). Root of the Eukaryota tree as inferred from combined maximum likelihood analyses of multiple molecular sequence data. Mol Biol Evol 22, 409420.

Biagini, G. A., Lloyd, D., Kirk, K. \& Edwards, M. R. (2000). The membrane potential of Giardia intestinalis. FEMS Microbiol Lett 192, 153-157.

Bradley, P. J., Lahti, C. J., Plümper, E. \& Johnson, P. J. (1997). Targeting and translocation of proteins into the hydrogenosome of the protist Trichomonas: similarities with mitochondrial protein import. EMBO J 16, 3484-3493.

Cavalier-Smith, T. (1987). Eukaryotes with no mitochondria. Nature 326, 332-333. 
Chan, K. W., Slotboom, D. J., Cox, S., Embley, T. M., Fabre, O., van der Giezen, M., Harding, M., Horner, D. S., Kunji, E. R. \& León-Avila, G. (2005). A novel ADP/ATP transporter in the mitosome of the microaerophilic human parasite Entamoeba histolytica. Curr Biol 15, 737-742.

Dandekar, T., Schuster, S., Snel, B., Huynen, M. \& Bork, P. (1999). Pathway alignment: application to the comparative analysis of glycolytic enzymes. Biochem J 343, 115-124.

Delgado-Viscogliosi, P., Brugerolle, G. \& Viscogliosi, E. (1996). Tubulin post-translational modifications in the primitive protist Trichomonas vaginalis. Cell Motil Cytoskeleton 33, 288-297.

Dolezal, P., Smíd, O., Rada, P., Zubácová, Z., Bursać, D., Suták, R., Nebesárová, J., Lithgow, T. \& Tachezy, J. (2005). Giardia mitosomes and trichomonad hydrogenosomes share a common mode of protein targeting. Proc Natl Acad Sci U S A 102, 10924-10929.

Ellis, J. E., Williams, R., Cole, D., Cammack, R. \& Lloyd, D. (1993). Electron transport components of the parasitic protozoon Giardia lamblia. FEBS Lett 325, 196-200.

Embley, T. M. (2006). Multiple secondary origins of the anaerobic lifestyle in eukaryotes. Philos Trans R Soc Lond B Biol Sci 361, 10551067.

Embley, T. M. \& Martin, W. (2006). Eukaryotic evolution, changes and challenges. Nature 440, 623-630.

Embley, T. M., van der Giezen, M., Horner, D. S., Dyal, P. L., Bell, S. \& Foster, P. G. (2003). Hydrogenosomes, mitochondria and early eukaryotic evolution. IUBMB Life 55, 387-395.

Emelyanov, V. V. (2001). Evolutionary relationship of Rickettsiae and mitochondria. FEBS Lett 501, 11-18.

Emelyanov, V. V. (2003a). Phylogenetic affinity of a Giardia lamblia cysteine desulfurase conforms to canonical pattern of mitochondrial ancestry. FEMS Microbiol Lett 226, 257-266.

Emelyanov, V. V. (2003b). Common evolutionary origin of mitochondrial and rickettsial respiratory chains. Arch Biochem Biophys 420, 130-141.

Emelyanov, V. V. (2003c). Mitochondrial connection to the origin of the eukaryotic cell. Eur J Biochem 270, 1599-1618.

Emelyanov, V. V. (2007). Constantin Merezhkowsky and the Endocaryotic hypothesis. In Origin of Mitochondria and Hydrogenosomes, pp. 201-237. Edited by W. F. Martin \& M. Müller. Berlin, Heidelberg: Springer-Verlag.

Fitzpatrick, D. A., Creevey, C. J. \& McInerney, J. O. (2006). Genome phylogenies indicate a meaningful alpha-proteobacterial phylogeny and support a grouping of the mitochondria with the Rickettsiales. Mol Biol Evol 23, 74-85.

Gill, E. E., Diaz-Triviño, S., Barberà, M. J., Silberman, J. D., Stechmann, A., Gaston, D., Tamas, I. \& Roger, A. J. (2007). Novel mitochondrion-related organelles in the anaerobic amoeba Mastigamoeba balamuthi. Mol Microbiol 66, 1306-1320.

Gray, M. W., Lang, B. F., Cedergren, R., Golding, G. B., Lemieux, C., Sankoff, D., Turmel, M., Brossard, N., Delage, E. \& other authors (1998). Genome structure and gene content in protist mitochondrial DNAs. Nucleic Acids Res 26, 865-878.

Gupta, R. S. (1998). Protein phylogenies and signature sequences: a reappraisal of evolutionary relationships among archaebacteria, eubacteria, and eukaryotes. Microbiol Mol Biol Rev 62, 1435-1491.

Hampl, V., Silberman, J. D., Stechmann, A., Diaz-Triviño, S., Johnson, P. J. \& Roger, A. J. (2008). Genetic evidence for a mitochondriate ancestry in the 'amitochondriate' flagellate Trimastix pyriformis. PLoS ONE 3, e1383.

Hampl, V., Hug, L., Leigh, J. W., Dacks, J. B., Lang, B. F., Simpson, A. G. \& Roger, A. J. (2009). Phylogenomic analyses support the monophyly of Excavata and resolve relationships among eukaryotic "supergroups". Proc Natl Acad Sci U S A 106, 3859-3864.

Hirt, R. P., Lal, K., Pinxteren, J., Warwicker, J., Healy, B., Coombs, G. H., Field, M. C. \& Embley, T. M. (2003). Biochemical and genetic evidence for a family of heterotrimeric G-proteins in Trichomonas vaginalis. Mol Biochem Parasitol 129, 179-189.

Hjort, K., Goldberg, A. V., Tsaousis, A. D., Hirt, R. P. \& Embley, T. M. (2010). Diversity and reductive evolution of mitochondria among microbial eukaryotes. Philos Trans R Soc Lond B Biol Sci 365, 713-727.

Horner, D. S., Hirt, R. P. \& Embley, T. M. (1999). A single eubacterial origin of eukaryotic pyruvate:ferredoxin oxidoreductase genes: implications for the evolution of anaerobic eukaryotes. Mol Biol Evol 16, 1280-1291.

Hug, L. A., Stechmann, A. \& Roger, A. J. (2010). Phylogenetic distributions and histories of proteins involved in anaerobic pyruvate metabolism in eukaryotes. Mol Biol Evol 27, 311-324.

Humphreys, M., Ralphs, J. R., Durrant, L. \& Lloyd, D. (1998). Confocal laser scanning microscopy of Trichomonads. Hydrogenosomes store calcium and show a membrane potential. Eur J Protistol 34, 356-362.

Keeling, P. J., Burger, G., Durnford, D. G., Lang, B. F., Lee, R. W., Pearlman, R. E., Roger, A. J. \& Gray, M. W. (2005). The tree of eukaryotes. Trends Ecol Evol 20, 670-676.

Laemmli, U. K. (1970). Cleavage of structural proteins during the assembly of the head of bacteriophage T4. Nature 227, 680-685.

Lill, R. \& Mühlenhoff, U. (2008). Maturation of iron-sulfur proteins in eukaryotes: mechanisms, connected processes, and diseases. Annu Rev Biochem 77, 669-700.

Lindmark, D. G. (1980). Energy metabolism of the anaerobic protozoon Giardia lamblia. Mol Biochem Parasitol 1, 1-12.

Lloyd, D., Ralphs, J. R. \& Harris, J. C. (2002a). Giardia intestinalis, a eukaryote without hydrogenosomes, produces hydrogen. Microbiology 148, 727-733.

Lloyd, D., Harris, J. C., Maroulis, S., Wadley, R., Ralphs, J. R., Hann, A. C., Turner, M. P. \& Edwards, M. R. (2002b). The "primitive" microaerophile Giardia intestinalis (syn. lamblia, duodenalis) has specialized membranes with electron transport and membrane-potential-generating functions. Microbiology 148, 1349-1354.

Maralikova, B., Ali, V., Nakada-Tsukui, K., Nozaki, T., van der Giezen, M., Henze, K. \& Tovar, J. (2010). Bacterial-type oxygen detoxification and iron-sulfur cluster assembly in amoebal relict mitochondria. Cell Microbiol 12, 331-342.

Martin, W. \& Müller, M. (1998). The hydrogen hypothesis for the first eukaryote. Nature 392, 37-41.

Mokranjac, D. \& Neupert, W. (2008). Energetics of protein translocation into mitochondria. Biochim Biophys Acta 1777, 758762.

Morrison, H. G., McArthur, A. G., Gillin, F. D., Aley, S. B., Adam, R. D., Olsen, G. J., Best, A. A., Cande, W. Z., Chen, F. \& other authors (2007). Genomic minimalism in the early diverging intestinal parasite Giardia lamblia. Science 317, 1921-1926.

Müller, M. (1993). The hydrogenosome. J Gen Microbiol 139, 28792889.

Müller, M. (2003). Energy metabolism. Part I: anaerobic protozoa. In Molecular Medical Parasitology, pp. 125-139. Edited by J. Marr, T. Nilsen \& R. Komunieki. London: Academic Press.

Pendergrass, W., Wolf, N. \& Poot, M. (2004). Efficacy of MitoTracker Green and CMXrosamine to measure changes in mitochondrial membrane potentials in living cells and tissues. Cytometry A 61, 162-169.

Pütz, S., Dolezal, P., Gelius-Dietrich, G., Bohacova, L., Tachezy, J. \& Henze, K. (2006). Fe-hydrogenase maturases in the hydrogenosomes of Trichomonas vaginalis. Eukaryot Cell 5, 579-586. 
Regoes, A., Zourmpanou, D., León-Avila, G., van der Giezen, M., Tovar, J. \& Hehl, A. B. (2005). Protein import, replication, and inheritance of a vestigial mitochondrion. J Biol Chem 280, 30557-30563.

Sánchez, L. B., Galperin, M. Y. \& Müller, M. (2000). Acetyl-CoA synthetase from the amitochondriate eukaryote Giardia lamblia belongs to the newly recognized superfamily of acyl-CoA synthetases (Nucleoside diphosphate-forming). J Biol Chem 275, 5794-5803.

Saraste, M. (1999). Oxidative phosphorylation at the fin de siècle. Science 283, 1488-1493.

Smíd, O., Matusková, A., Harris, S. R., Kucera, T., Novotný, M., Horváthová, L., Hrdý, I., Kutejová, E., Hirt, R. P. \& other authors (2008). Reductive evolution of the mitochondrial processing peptidases of the unicellular parasites Trichomonas vaginalis and Giardia intestinalis. PLoS Pathog 4, e1000243.

Stefanic, S., Palm, D., Svärd, S. G. \& Hehl, A. B. (2006). Organelle proteomics reveals cargo maturation mechanisms associated with Golgi-like encystation vesicles in the early-diverged protozoan Giardia lamblia. J Biol Chem 281, 7595-7604.

Sutak, R., Dolezal, P., Fiumera, H. L., Hrdy, I., Dancis, A., DelgadilloCorrea, M., Johnson, P. J., Müller, M. \& Tachezy, J. (2004). Mitochondrial-type assembly of FeS centers in the hydrogenosomes of the amitochondriate eukaryote Trichomonas vaginalis. Proc Natl Acad Sci U S A 101, 10368-10373.

Tachezy, J., Sánchez, L. B. \& Müller, M. (2001). Mitochondrial type iron-sulfur cluster assembly in the amitochondriate eukaryotes Trichomonas vaginalis and Giardia intestinalis, as indicated by the phylogeny of IscS. Mol Biol Evol 18, 1919-1928.
Takaya, N., Suzuki, S., Kuwazaki, S., Shoun, H., Maruo, F., Yamaguchi, M. \& Takeo, K. (1999). Cytochrome p450nor, a novel class of mitochondrial cytochrome $\mathrm{P} 450$ involved in nitrate respiration in the fungus Fusarium oxysporum. Arch Biochem Biophys 372, 340-346.

Tielens, A. G. \& Van Hellemond, J. J. (1998). The electron transport chain in anaerobically functioning eukaryotes. Biochim Biophys Acta 1365, 71-78.

Tovar, J., León-Avila, G., Sánchez, L. B., Sutak, R., Tachezy, J., van der Giezen, M., Hernández, M., Müller, M. \& Lucocq, J. M. (2003). Mitochondrial remnant organelles of Giardia function in ironsulphur protein maturation. Nature 426, 172-176.

Townson, S. M., Upcroft, J. A. \& Upcroft, P. (1996). Characterisation and purification of pyruvate: ferredoxin oxidoreductase from Giardia duodenalis. Mol Biochem Parasitol 79, 183-193.

van Grinsven, K. W., Rosnowsky, S., van Weelden, S. W., Pütz, S., van der Giezen, M., Martin, W., van Hellemond, J. J., Tielens, A. G. \& Henze, K. (2008). Acetate: succinate CoA-transferase in the hydrogenosomes of Trichomonas vaginalis: identification and characterization. J Biol Chem 283, 1411-1418.

Van Hellemond, J. J., Opperdoes, F. R. \& Tielens, A. G. (1998). Trypanosomatidae produce acetate via a mitochondrial acetate: succinate CoA transferase. Proc Natl Acad Sci U S A 95, 3036-3041.

Williams, K. P., Sobral, B. W. \& Dickerman, A. W. (2007). A robust species tree for the alphaproteobacteria. J Bacteriol 189, 4578-4586.

Edited by: L. J. Knoll 